\title{
Formulation and pharmaceutical development of quetiapine fumarate sustained release matrix tablets using a $\mathrm{QbD}$ approach
}

\author{
ALEXANDRU GAVAN \\ ALINA PORFIRE* \\ CRISTINA MARINA \\ IOAN TOMUTA \\ University of Medicine and Pharmacy \\ "Iuliu Hatieganu" \\ Faculty of Pharmacy \\ Department of Pharmaceutical \\ Technology \\ and Biopharmaceutics, Cluj-Napoca \\ Romania
}

\begin{abstract}
The main objective of the present study was to apply QbD methodology in the development of once-a-day sustained release quetiapine tablets. The quality target product profile (QTPP) was defined after the pharmaceutical properties and kinetic release of the innovator product, Seroquel XR $200 \mathrm{mg}$. For the D-optimal experimental design, the level and ratio of matrix forming agents and the type of extragranular diluent were chosen as independent inputs, which represented critical formulation factors. The critical quality attributes (CQAs) studied were the cumulative percentages of quetiapine released after certain time intervals. After the analysis of the experimental design, optimal formulas and the design space were defined. Optimal formulas demonstrated zero-order release kinetics and a dissolution profile similar to the innovator product, with $\mathrm{f}_{2}$ values of 74.53 and 83.74. It was concluded that the QbD approach allowed fast development of sustained release tablets with similar dissolution behavior as the innovator product.
\end{abstract}

Keywords: quality by design, design of experiments, sustained release, hydrophilic matrix, quetiapine

In the past years, the main goal of antipsychotic drug development has been to develop molecules with higher efficacy and fewer side effects usually associated with the classic antipsychotic medications. The newly developed agents, called atypical antipsychotics, are successfully used in the treatment of both positive and negative symptoms of schizophrenia and are associated with fewer neurological and endocrine side effects compared to older medications (1). Quetiapine is a recently introduced atypical antipsychotic, which has an excellent risk/benefit ratio and is indicated as the first-line option for the treatment of psychotic disorder manifestations and schizophrenia (2). Quetiapine is available as fumarate salt in immediate as well as in sustained released formulations, the innovative product being Seroquel. The sustained release formulation was introduced several years ago and its primary objective is to release the drug slowly over an extended

\footnotetext{
*Correspondence; e-mail: aporfire@umfcluj.ro
} 
A. Gavan et al:: Formulation and pharmaceutical development of quetiapine fumarate sustained release matrix tablets using a QbD approach, Acta Pharm. 67 (2017) 53-70.

period of time, ensuring safety and improving the efficacy of the medical treatment as well as patient compliance (3).

One of the most popular delivery systems for oral sustained release dosage forms are hydrophilic matrix tablets, which are widely accepted because of their biopharmaceutical advantages over other types of dosage forms. The reasons behind the popularity of hydrophilic matrixes are that they offer precise drug release modulation as a result of hydration of non-ionic cellulose ethers, cost effectiveness, and the capacity to provide a prolonged and constant therapeutic effect $(4,5)$. Although developing a hydroxypropyl methylcellulose (HPMC) matrix formulation may seem simple, a large number of variables need to be considered for a valid formulation with the desired release kinetics. The release rate from the matrix is influenced by factors such as polymer type and level, drug dose and solubility, polymer-drug ratio, diluent type and level, polymer-diluent ratio and porosity of the matrix (6). Pharmacokinetic theory suggests that the ideal release of the drug from a sustained release dosage form, without fluctuations of drug levels in blood stream, is the zero order profile. In this situation, the drug is released at the same slow rate throughout the entire period, and once steady state is achieved, drug concentration is constant as long as absorption persists $(7,8)$. The strategy of blending high and low viscosity grades of HPMC has been used before for the development of hydrophilic matrix formulations with zero order release profiles and for reducing the drug release variability, providing uniform drug levels (9).

Conventional optimization methods where one factor is optimized at a time constitute the main shortcoming, since they do not take into account potential interactions between factors, which can lead to a failure in identification of the optimal combination. The recently proposed Quality by Design $(\mathrm{QbD})$ regulatory initiative represents a highly systematic approach implementing the Design of Experiments (DoE) for finding the optimal product and process characteristics. DoE provides the largest amount of information from the least number of experimental runs by systematic variation of the factors and simultaneous evaluation of the effects of multiple variables. Quality assurance has changed from the need to show that the final product meets predefined specifications to a new situation where it needs to be demonstrated that the product is controlled within a well-defined design space $(10,11)$. The design space is defined as an established multidimensional range of input variables (e.g., formulation factors) and process parameters demonstrated to provide assurance of quality (12).

Use of in vitro drug dissolution values as DoE output variables has been recognized as an important strategy for drug development. Drug release from immediate and sustained oral dosage forms is described by several kinetic equations. Interpretation of output variables representing in vitro release values is realized with the help of mathematical polynomial equations that convert the dissolution profiles into parameters relating to the pharmaceutical characteristics of the dosage form $(13,14)$.

The aim of this study was to apply a QbD approach in order to develop once-a-day sustained release 200-mg quetiapine tablets. A quadratic D-optimal experimental design was used to evaluate the effect of matrix-forming polymer (HPMC) percentage and filler type on the cumulative ratio of drug released at different time intervals for a period of 24 hours, and to define the optimal formulations. The optimization procedure would help define the design space and set up formulation parameters for the preparation of controlled release tablets with predictable properties. 
A. Gavan et al:: Formulation and pharmaceutical development of quetiapine fumarate sustained release matrix tablets using a QbD approach, Acta Pharm. 67 (2017) 53-70.

\section{EXPERIMENTAL}

\section{Materials}

Quetiapine fumarate was purchased from Menadiona, Spain. Lactose monohydrate - Pharmatose 150M (DFE Pharma, Germany) was used as a diluent and polyvinylpyrrolidone - Kollidon 30 (BASF, Germany) as a binder in the granulation step. Two types of HPMC with different viscosity grades - Methocel K100 Premium LV CR and K4M Premium CR (Colorcon, United Kingdom) were used as matrix-forming excipients. Lactose monohydrate - Tablettose 80 (Meggle, Germany), microcrystalline cellulose - Avicel PH102 (FMC BioPolymer, USA), fumed silica - Aerosil 200 (Röhm, Germany) and magnesium stearate - Kemilub EM-F (Undesa, Spain) were used as extragranular excipients.

\section{QbD steps}

The first step in a QbD type study is to define the Quality Target Product Profile (QTPP) and to identify the potential Critical Quality Attributes (CQAs) based on it. QTPP was defined by the International Conference on Harmonization (ICH) Q8 as ", prospective summary of the quality characteristics of a drug product that ideally will be achieved to ensure the desired quality, taking into account safety and efficacy of the drug product" (15) and it represents the basis for the design of a generic product (16). The QTPP was set based on literature specifications for sustained release oral tablets and on the results obtained after analyzing the innovative quetiapine extended release product, Seroquel XR. The developed product should contain $200 \mathrm{mg}$ quetiapine that will be released from a hydrophilic matrix over 24 hours, similarly to the innovator product. According to the European Pharmacopeia specifications, the tablets should be free of any visual defects, the quetiapine identification should be positive and the active pharmaceutical ingredient (API) content variation should not exceed $5 \%$. The mass of the tablets should be uniform, with variations under $5 \%$, friability should be under $1 \%(\mathrm{~m} / \mathrm{m})$ and the crushing resistance over $150 \mathrm{~N}$ in order to assure tablet integrity (17). The dissolution profile should be similar to the profile of the innovator product; in this way, similar absorption kinetics should be achieved by controlling the kinetic drug release from the dosage form.

Also, according to the ICH Q8, the CQA is ", a quality attribute (a physical, chemical, biological or microbiological property of characteristic) that must be controlled (directly or indirectly) to ensure that the product meets its intended safety, efficacy, stability and performance" (15). The most significant CQAs that need to be considered in the pharmaceutical development of sustained release formulations are the dissolution release profile and kinetic drug release. According to the literature, the levels of matrix forming agents and the type of extragranular diluent are the potential risk factors that might influence the CQAs of a sustained release formulation; this is the reason why these factors were chosen as variables in this study.

\section{Experimental design}

To be able to understand the product and refine the formulation of sustained release hydrophilic matrix tablets, we have chosen to use a full factorial D-optimal experimental design with one qualitative and two quantitative factors and five levels, using Modde 11.0 software (Umetrics, Sweden). 
Preliminary studies were performed to select the starting formulations, and it was observed that the desired kinetic release profile could only be obtained by using a combination of two matrix forming excipients with different viscosities. In the formulation, the main matrix forming excipient used was Methocel K100, blended in various proportions with a higher viscosity polymer, Methocel K4M. In the DoE, the first chosen formulation factor was the total percent of matrix forming excipient, the second quantitative factor being the proportion of Methocel K4M to the total quantity of matrix forming excipient used for each formulation. The selected qualitative factor was the type of extragranular diluent. The responses studied were the cumulative percentages of quetiapine released at different time intervals. The levels of variation for the independent and dependent variables are presented in Table I. The effects of independent variables over the measured responses were evaluated by the analysis of coefficients and contour plots. Based on these results, the design space and optimal formulations were calculated by the Modde software.

In general, the experimental design region defined in the $\mathrm{QbD}$ approach illustrates the whole range of interactions between the chosen inputs and the studied responses, whereas the design space is defined by ICH Q8 as ",the multidimensional combination and interaction

Table I. Symbols and levels of variables used in the design

\begin{tabular}{|c|c|c|c|c|}
\hline \multirow{2}{*}{ Variables } & \multirow[t]{2}{*}{ Symbol } & \multicolumn{3}{|c|}{ Levels } \\
\hline & & Low & \multicolumn{2}{|r|}{ High } \\
\hline \multicolumn{5}{|l|}{ Independent variables (factors) } \\
\hline Total HPMC (\%) & $X_{1}$ & 30 & \multicolumn{2}{|r|}{45} \\
\hline HPMC K4M from the total HPMC (\%) & $X_{2}$ & 0 & \multicolumn{2}{|r|}{50} \\
\hline Type of extragranular diluent & $X_{3}$ & $\begin{array}{c}\text { Lactose } \\
\text { monohydrate }\end{array}$ & \multicolumn{2}{|r|}{$\begin{array}{l}\text { Microcrystalline } \\
\text { cellulose }\end{array}$} \\
\hline \multicolumn{2}{|l|}{ Dependent variables (responses) } & \multicolumn{3}{|c|}{ CQAs } \\
\hline & & Minimum & Target & Maximum \\
\hline Cumulative $\%$ of quetiapine released after $1 \mathrm{~h}$ & $Y_{1}$ & 3 & 5 & 7 \\
\hline Cumulative \% of quetiapine released after $2 \mathrm{~h}$ & $Y_{2}$ & 6 & 10 & 12 \\
\hline Cumulative \% of quetiapine released after $3 \mathrm{~h}$ & $Y_{3}$ & 10 & 14 & 17 \\
\hline Cumulative $\%$ of quetiapine released after $4 \mathrm{~h}$ & $Y_{4}$ & 14 & 18 & 22 \\
\hline Cumulative \% of quetiapine released after $6 \mathrm{~h}$ & $Y_{5}$ & 23 & 28 & 33 \\
\hline Cumulative $\%$ of quetiapine released after $8 \mathrm{~h}$ & $Y_{6}$ & 32 & 36 & 43 \\
\hline Cumulative $\%$ of quetiapine released after $10 \mathrm{~h}$ & $Y_{7}$ & 41 & 46 & 53 \\
\hline Cumulative $\%$ of quetiapine released after $12 \mathrm{~h}$ & $Y_{8}$ & 50 & 56 & 62 \\
\hline Cumulative \% of quetiapine released after $18 \mathrm{~h}$ & $Y_{9}$ & 70 & 79 & 85 \\
\hline Cumulative $\%$ of quetiapine released after $24 \mathrm{~h}$ & $Y_{10}$ & 84 & 93 & 100 \\
\hline
\end{tabular}


of input variables that have been demonstrated to provide assurance of quality" (15). In regulatory terms, changes inside the design space do not need to be considered as changes, only changes outside this space should initiate post approval procedures (18). Further in this direction, a proven acceptable range (PAR) was defined inside the design space. The PAR is explained by ICH Q8 as ,, a characterized range of a process parameter for which operation within this range, while keeping other parameters constant, will result in producing a material meeting relevant quality criteria" (15). This acceptable range is defined by a design space hypercube, which represents the largest possible regular hypercube that can be inserted into the irregular design space and shows the volume in which all factor combinations can be used without compromising the product's CQAs (19). After the determination of the design space and PAR, the optimal formulations were determined by defining combinations of factor values that predict a result as close as possible to the target values of all responses (20).

\section{Tablet preparation}

In order to obtain good quality hydrophilic matrix tablets, a preliminary granulation step was required; the formulation of the granules and the granulation parameters were established during preliminary studies (data not shown). Quetiapine fumarate (82\%) and lactose monohydrate (15\%) were granulated in an Aeromatic Strea 1 fluidized bed granulator (GEA, Switzerland) using a polyvinylpyrrolidone solution (3\%) as binding agent. A quantity of granules corresponding to $200 \mathrm{mg}$ of quetiapine/tablet unit was subsequently mixed for 5 minutes in a polyethylene bag together with the extragranular excipients. The formulation is given in Table II. Tablets were compressed from the mixes using an eccentric tablet press (Korsch EK-0, Germany) equipped with a $13 \mathrm{~mm}$, round, flat and plain punch. The compressing machine was adjusted so that the manufactured tablets had an average weight of $\sim 640 \mathrm{mg}$ and a crushing strength greater than $50 \mathrm{~N}$.

\section{Tablet evaluation}

Standard evaluation tests specified for tablets in the European Pharmacopoeia were performed (17). Mass variation was determined by weighting 20 tablets individually, the

Table II. Formulation of the hydrophilic matrix tablets

\begin{tabular}{lcc}
\hline Ingredient & $\begin{array}{c}\text { Percent in formulation } \\
(\%)\end{array}$ & $\begin{array}{c}\text { Quantity for a single tablet } \\
(\mathrm{mg})\end{array}$ \\
\hline Quetiapine fumarate granulate & 44.7 & 286.1 \\
HPMC K100 & $30-45$ & $96-288$ \\
HPMC K4M & $0-22,5$ & $0-144$ \\
Lactose monohydrate / Microcrystalline & $9.3-24.3$ & $59.5-155.5$ \\
cellulose & 0.5 & 3.20 \\
Fumed silica & 0.5 & 3.20 \\
Magnesium stearate & 100 & 640 \\
Total & & \\
\hline
\end{tabular}


average mass was calculated and percent of variation was calculated. Hardness was evaluated on 6 tablets from each series using a Dr. Schleuniger Pharmaton 6D hardness tester (Pharmaton, Switzerland) and friability was evaluated on 10 tablets using an Erweka TA10 friabilator (Erweka, Germany) set at 100 rotations.

In vitro dissolution studies for the developed tablets, containing $200 \mathrm{mg}$ quetiapine, were performed using a PharmaTest PTWS 100 dissolution tester (PharmaTest, Germany) applying the basket method (European Pharmacopoeia apparatus 1) at $100 \mathrm{rpm}$ in $900 \mathrm{~mL} 0.05 \mathrm{~mol} \mathrm{~L}^{-1}$ phosphate buffer $\mathrm{pH} 6.8$, maintained at $37 \pm 0.5^{\circ} \mathrm{C} .5 \mathrm{~mL}$ samples were withdrawn through $10 \mu \mathrm{m}$ cannula filters at $1,2,3,4,6,8,10,12,18$ and $24 \mathrm{~h}$. In order to maintain a constant volume throughout the experiment, each taken sample was replaced with the same volume of fresh medium. Dissolution studies were run in triplicate for each formula and the absorbance was measured on 1:5 diluted samples at $246 \mathrm{~nm}$ using a Specord 200 Plus UV/Vis Spectrophotometer (Analytik Jena, Germany). Cumulative drug release was calculated using an equation determined from Lambert Beer's calibration curve in the linearity range of $0-50 \mu \mathrm{g} \mathrm{mL}^{-1}$. For each sampling point, mean and standard deviations were calculated.

\section{Release kinetics}

To evaluate the release profiles of the formulations, several release profiles were tested, such as zero order, first order (21, 22), Higuchi (23), Hixon-Crowell (24) and KorsmeyerPeppas (25). Mathematical models were fitted one at a time to individual dissolution data, using the SigmaPlot 11.0 (Systat software, USA) software. For each model, regression analyses were applied to obtain the release constant $k$, correlation coefficient $R^{2}$ and the Akaike Information Criterion (AIC). The use of AIC helped compare the competing models; a lower value of this criterion means a better model fit.

\section{RESULTS AND DISCUSSIONS}

\section{Preparation and evaluation of tablet formulations}

All the batches were produced under similar conditions to avoid processing variables and the hydrophilic matrix tablets from each batch were analyzed according to the European Pharmacopoeia methods for average mass, mass uniformity, crushing resistance and friability (17). According to the obtained results, all prepared formulations had an average mass of $640 \pm 7 \mathrm{mg}$ with the intra-batch weight variability lower than $1.9 \%$. The tablets showed a high crushing resistance between 50 and $180 \mathrm{~N}$ and very low friability under $1 \%$.

Thus, all the tablet batches demonstrated very good pharmaceutical properties, which complied with the European Pharmacopoeia requirements, proving that the different proportions of hydrophilic polymers and diluents used in the formulations did not influence the uniform filling of the dyes during the tableting step.

\section{CQAs of sustained release formulations}

Critical Quality Attributes (CQAs) are physical, chemical, biological, or microbiological properties or characteristics that should be within an appropriate limit, range, or dis- 
A. Gavan et al.: Formulation and pharmaceutical development of quetiapine fumarate sustained release matrix tablets using a QbD approach, Acta Pharm. 67 (2017) 53-70.

Table III. Experimental design matrix of factors and responses

\begin{tabular}{|c|c|c|c|c|c|c|c|}
\hline Experiment code & $X_{1}$ & $X_{2}$ & $X_{3}$ & & $Y_{1}$ & $Y_{2}$ & $Y_{3}$ \\
\hline $\mathrm{N} 1$ & 30 & 0 & lactose & & 11 & 21 & 31 \\
\hline N2 & 45 & 0 & lactose & & 6 & 13 & 20 \\
\hline N3 & 30 & 50 & lactose & & 9 & 16 & 22 \\
\hline N4 & 45 & 50 & lactose & & 5 & 8 & 12 \\
\hline N5 & 30 & 33.3 & lactose & & 9 & 16 & 23 \\
\hline N6 & 45 & 16.7 & lactose & & 6 & 11 & 17 \\
\hline N7 & 35 & 0 & lactose & & 11 & 21 & 30 \\
\hline N8 & 40 & 50 & lactose & & 4 & 8 & 12 \\
\hline N9 & 37.5 & 25 & lactose & & 7 & 13 & 19 \\
\hline N10 & 30 & 0 & cellulose & & 10 & 18 & 27 \\
\hline N11 & 45 & 0 & cellulose & & 6 & 13 & 19 \\
\hline N12 & 30 & 50 & cellulose & & 8 & 13 & 17 \\
\hline N13 & 45 & 50 & cellulose & & 5 & 8 & 11 \\
\hline N14 & 30 & 16.7 & cellulose & & 11 & 18 & 24 \\
\hline N15 & 45 & 33.3 & cellulose & & 4 & 8 & 12 \\
\hline N16 & 40 & 0 & cellulose & & 8 & 14 & 21 \\
\hline N17 & 35 & 50 & cellulose & & 6 & 10 & 14 \\
\hline N18 & 37.5 & 25 & cellulose & & 8 & 13 & 18 \\
\hline N19 & 37.5 & 25 & cellulose & & 6 & 11 & 16 \\
\hline \multirow[t]{2}{*}{ N20 } & 37.5 & 25 & cellulose & & 6 & 11 & 16 \\
\hline & $Y_{4}$ & $Y_{5}$ & $Y_{6}$ & $Y_{7}$ & $Y_{8}$ & $Y_{9}$ & $Y_{10}$ \\
\hline N1 & 40 & 58 & 72 & 82 & 90 & 90 & 90 \\
\hline N2 & 26 & 38 & 50 & 60 & 69 & 88 & 89 \\
\hline N3 & 28 & 39 & 47 & 53 & 60 & 77 & 87 \\
\hline N4 & 16 & 23 & 30 & 34 & 39 & 52 & 62 \\
\hline N5 & 30 & 44 & 54 & 64 & 73 & 91 & 98 \\
\hline N6 & 23 & 34 & 43 & 54 & 63 & 82 & 94 \\
\hline N7 & 37 & 52 & 64 & 76 & 85 & 95 & 97 \\
\hline N8 & 15 & 23 & 29 & 35 & 40 & 54 & 66 \\
\hline N9 & 25 & 35 & 44 & 51 & 58 & 80 & 93 \\
\hline N10 & 35 & 49 & 62 & 72 & 83 & 91 & 92 \\
\hline N11 & 25 & 37 & 47 & 58 & 66 & 87 & 93 \\
\hline N12 & 21 & 29 & 34 & 39 & 44 & 57 & 67 \\
\hline N13 & 14 & 20 & 25 & 28 & 31 & 43 & 52 \\
\hline N14 & 30 & 40 & 48 & 55 & 62 & 84 & 95 \\
\hline N15 & 17 & 24 & 31 & 38 & 43 & 57 & 71 \\
\hline N16 & 28 & 40 & 51 & 61 & 69 & 90 & 97 \\
\hline N17 & 17 & 24 & 31 & 36 & 40 & 52 & 62 \\
\hline N18 & 23 & 33 & 42 & 49 & 57 & 78 & 96 \\
\hline N19 & 21 & 30 & 39 & 46 & 54 & 74 & 90 \\
\hline N20 & 21 & 30 & 39 & 46 & 53 & 72 & 90 \\
\hline
\end{tabular}

$X_{1}-X_{3}$ - independent variables (factors) according to Table II.

$Y_{1}-Y_{10}-$ dependent variables (responses), according to Table II. 
A. Gavan et al:: Formulation and pharmaceutical development of quetiapine fumarate sustained release matrix tablets using a QbD approach, Acta Pharm. 67 (2017) 53-70.

tribution to ensure the desired product quality, the values that need to be well controlled in order to ensure the efficacy and performance of the designed product (15).

In order to rapidly define a formulation with similar release kinetics as the target product profile, the D-optimal experimental design was applied. After preliminary studies and based on the literature, it was established that the following formulation factors would have the main role in assuring the correct kinetic drug release from the hydrophilic matrix, the amount and proportion of HPMC and the type of extragranular diluent. Therefore, the studied critical formulation factors were the total percent of HPMC $\left(X_{1}\right)$, proportion of the high viscosity HPMC used, reported to the total quantity of matrix forming excipient $\left(X_{2}\right)$ and the type of extragranular diluent $\left(X_{3}\right)$. For an extended release formulation, the most significant parameters that assure the product performance and efficacy are the dissolution profile defined by cumulative percentages of the drug released at different time intervals. Thus, the responses of the experimental design, namely, cumulative percentages of the drug released at different time intervals $\left(Y_{1}\right.$ to $\left.Y_{10}\right)$, were established as CQAs. The accepted intervals for the CQAs were calculated considering the dissolution profile of the innovative product Seroquel XR and are presented in Table I.

\section{Experimental design analysis. Summary of fit}

The experimental design matrix generated by the Modde software and completed with the responses obtained after performing all experimental runs is shown in Table III. The results show that the kinetic release of quetiapine was influenced by the formulation factors studied in the experimental design.

The multiple linear regression (MLR) fitting of the experimental data with the experimental design was evaluated using the following statistical parameters: $R^{2}, Q^{2}$ and ANOVA test. $R^{2}$ represents the goodness of fit and describes the fraction of variation of the response explained by the model, while $\mathrm{Q}^{2}$ represents the goodness of prediction and estimates the prediction capacities of the model. The values of $R^{2}$ and $Q^{2}$ are between 0 and 1; for a model to pass the diagnostic test, both parameters should have high values indicating a good model with very high predictive power. The difference between the two should be no more than $0.2-0.3$, higher differences indicating an inappropriately selected model. Together, $R^{2}$ and $\mathrm{Q}^{2}$ are the most reliable statistical parameters to describe the validity of a model (26).

A summary of fit plot was generated for all responses obtained after fitting and statistical parameter calculation using the data obtained from the experimental design. The selected model showed excellent quality, responses from $Y_{2}$ to $Y_{10}$ had values of over 0.93 for $R^{2}$, over 0.86 for $Q^{2}$. Slightly lower, but still excellent, values were registered for $Y 1\left(R^{2}\right.$ of around 0.87 and $Q^{2}$ of 0.73 ).

Another important diagnostic test for model validity is the ANOVA test (analysis of variance), which shows whether the variance of the results is determined by modifications in the formulation factors or represents a variance determined by experimental errors (26). For all dependent variables, the $p$ values of the ANOVA test were lower than 0.001 for the model and greater than 0.31 for the lack of fit. These results show that the quadratic model is statistically good, implying a significant influence of the factors on the responses, and that the model has no lack of fit. 
A. Gavan et al.: Formulation and pharmaceutical development of quetiapine fumarate sustained release matrix tablets using a QbD approach, Acta Pharm. 67 (2017) 53-70.

\section{Experimental design analysis. Evaluation of variables affecting kinetic drug release}

The in vitro release data over 24 hours of quetiapine from the developed hydrophilic matrix tablets at different dissolution time intervals are displayed in Table III. The MLR fitting of the experimental data and the regression coefficients were determined using the following equation:

$$
Y_{n}=b_{0}+b_{1} X_{1}+b_{2} X_{2}+b_{3} X_{3}+b_{13} X_{1} X_{3}+b_{22} X_{2}^{2}
$$

where $Y_{n}$ is the dependent variable; $b_{0}$ is the model constant; $b_{1}, b_{2}, b_{3}$ are linear coefficients; $b_{13}$ is an interaction coefficient between two factors; $b_{22}$ is a quadratic coefficient and $X_{1}, X_{2}$, $X_{3}$ are the coded levels of independent variables. The equation coefficients used to fit the data to the quadratic model represent the influence of the studied factors and of the interactions between factors on the responses.

Two and three dimensional plots were formed, based on the model, for evaluation of the responses, in order to assess the change of the response surface. Also, the relationship between formulation factors (independent variables) and responses (dependent variables) can be further understood from these plots $(19,27)$.

The influence of formulation factors on the most relevant responses $\left(Y_{2}, Y_{4}, Y_{6}, Y_{8}, Y_{9}\right.$ and $\left.Y_{10}\right)$ is presented as scaled and centered coefficients in Figure 1 and as the response surface plot in Figure $2(a, b)$, for the formulations with lactose as diluent (N1-N9) and for the formulations with cellulose as extragranular diluent (N10-N20), respectively. The same tendencies regarding the influence of formulation factors were found for the other responses analyzed in the experimental design $\left(Y_{1}, Y_{3}, Y_{5}\right.$, and $\left.Y_{7}\right)$. Results of the coefficient data analysis indicate that the amount of the matrix forming agent $\left(X_{1}\right)$ and the proportion of Methocel K4M blended with Methocel K100 $\left(X_{2}\right)$ had the most significant influence on drug release.For this type of formulation, erosion is the principal mechanism of drug release and the blending of two polymers with different viscosities was an alternative to obtain a hydrophilic matrix that allowed the desired extended drug release over 24 hours. In the developed formulations, the main component of the matrix forming polymer was the low-viscosity grade HPMC, which helped maintain a consistent erosion of the matrix, allowing a constant drug release over the whole desired period of time. This effect is shown in the coefficient plot by the relative constant negative value of the coefficient of $X_{1}$ (values between 3.5 and 8 ) at all dissolution points (Fig. 1). Consistent erosion of the matrix assured by this polymer is represented on the contour plots by the linear relationship between the coefficient of $X_{1}$ and the responses, observed over the whole period of time studied (Fig. 2). Addition of the higher viscosity polymer, Methocel K4M, increased the gel strength and assured integrity of the matrix over a longer period of time. This means that drug release decreases with an increase of the amount of high viscosity polymer (9). The effect is also described by the evolution of the coefficient plot; influence of the coefficient factor $X_{2}$ increases with time, being smaller in the first hours and growing with time. In this case, the existence of the $X_{2}{ }^{*} X_{2}$ term must be indicated, which is insignificant in the first 12 hours of the dissolution $\left(Y_{2}\right.$ to $\left.Y_{8}\right)$, but its intensity increases sharply for the last two dissolution points, after 18 and 24 hours, respectively $\left(Y_{9}\right.$ and $\left.Y_{10}\right)$, points at which it has a significant influence on the responses. The same phenomenon is displayed on the contour plot, the relationship between factor $X_{2}$ and the responses $Y_{2}$ to $Y_{8}$ is linear, but becomes 
A. Gavan et al.: Formulation and pharmaceutical development of quetiapine fumarate sustained release matrix tablets using a QbD approach, Acta Pharm. 67 (2017) 53-70.

non-linear in the second half of the dissolution period, indicating the quadratic nature of the relationship between factor $X_{2}$ and the responses $Y_{9}$ or $Y_{10}$.

The difference in drug release between formulations containing lactose monohydrate and microcrystalline cellulose as extragranular diluents can be mainly attributed to the
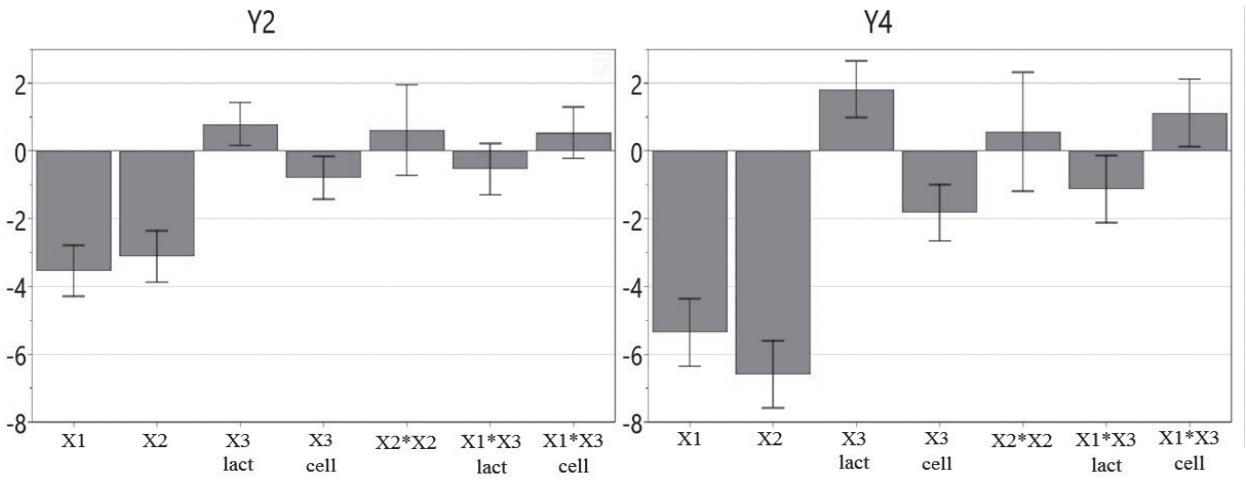

Y6

Y8
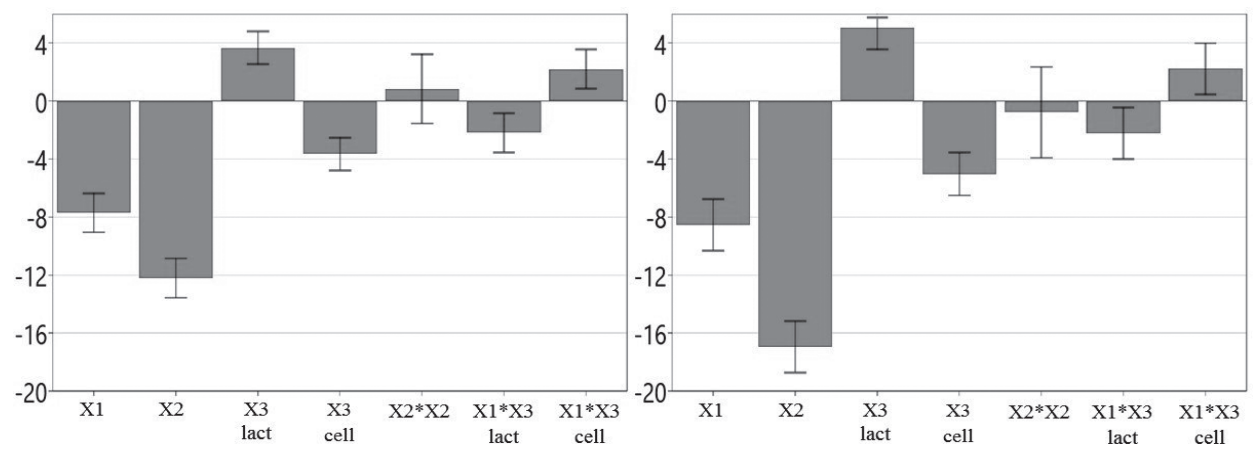

Y9

Y10
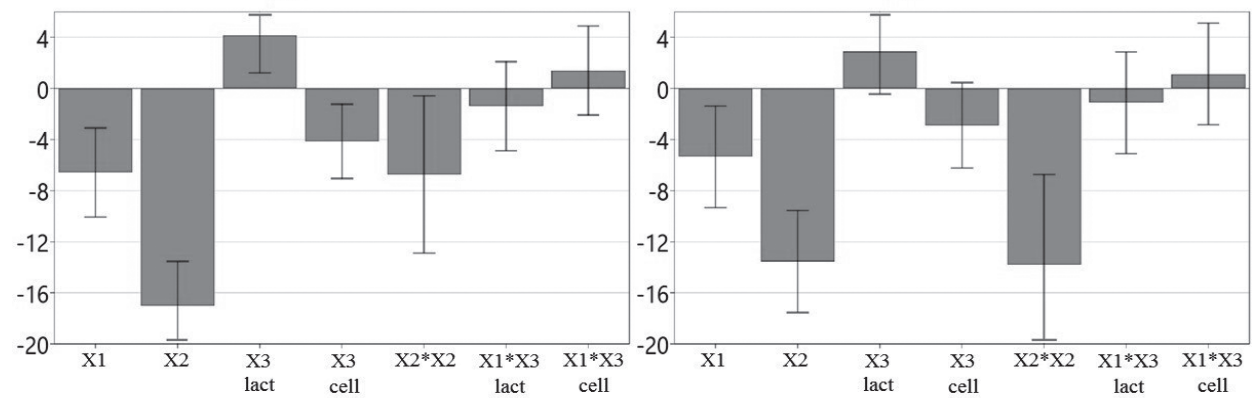

Fig. 1. Influence of formulation factors on the in vitro drug release at different dissolution time points, presented as scaled and centered coefficient plots. $X_{1}$ - total HPMC percent; $X_{2}-$ HPMC K4M proportion of the total HPMC percent; $X_{3}$ - type of extragranular diluent (lactose, cellulose); $Y_{2}, Y_{4}, Y_{6}, Y_{8}$, $Y_{9}, Y_{10}$ - dependent variables according to Table II. 


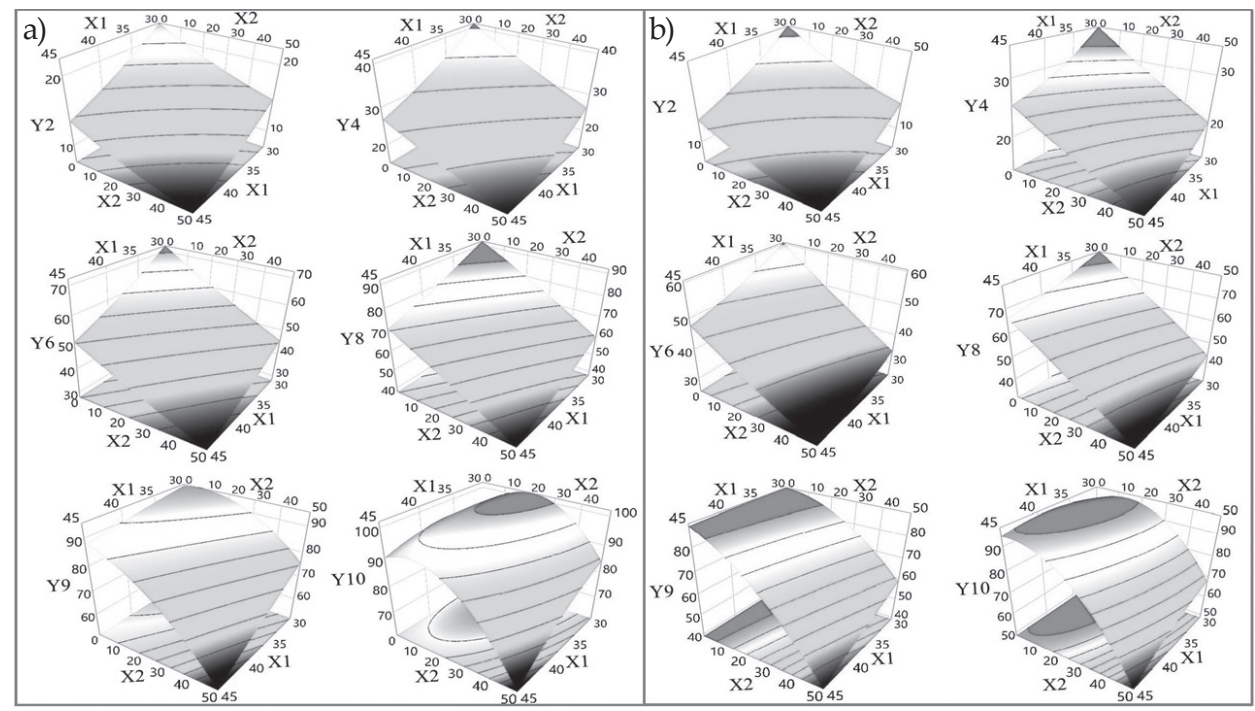

Fig. 2. Influence of formulation factors on the in vitro drug release at different dissolution time points for formulations with: a) lactose monohydrate and b) microcrystalline cellulose as diluents, represented as contour plots. $X_{1}$ - total HPMC percent; $X_{2}$ - HPMC K4M proportion of the total HPMC percent; $Y_{2}, Y_{4}, Y_{6}, Y_{8}, Y_{9}, Y_{10}$ - dependent variables according to Table II.

differences in solubility of the two excipients. Lactose will dissolve and will increase water availability within the gel structure of the hydrophilic matrix, favoring a diffusion mechanism besides the main erosion mechanism and in this way increasing drug release (28). On the other hand, microcrystalline cellulose will slow down the release of the active substance. This can be explained by the fact that, in the added proportions (10-25\%), cellulose has some binding properties that will harden the tablet and slow down matrix erosion (29). The two studied diluents influence drug release in opposite directions, but with approximately equal intensities. Their influence increases gradually and becomes constant after the first 4 hours of the dissolution. No significant interactions were registered between the studied formulation factors; the quality of the model was enhanced if these coefficients were discarded.

\section{Design space and formulation optimization}

In order to obtain a sustained release dosage form with constant drug release over 24 hours, the experimental formulations were compared with the dissolution profile of the original controlled release dosage form available on the market, namely, Seroquel XR 200 $\mathrm{mg}$. The release profile of the reference dosage form was analyzed and the QTPP was established. From the initial investigation domain, a design space was identified, where all the CQAs specifications should be fulfilled. Using the design space explorer function from the optimization module of the Modde software, the design space shown in Fig. 3 was developed by applying a series of limitations indicated in Table I, setting the CQAs to either a minimum or a maximum. Given that one input was qualitative (the type of extragranular 
A. Gavan et al.: Formulation and pharmaceutical development of quetiapine fumarate sustained release matrix tablets using a QbD approach, Acta Pharm. 67 (2017) 53-70.

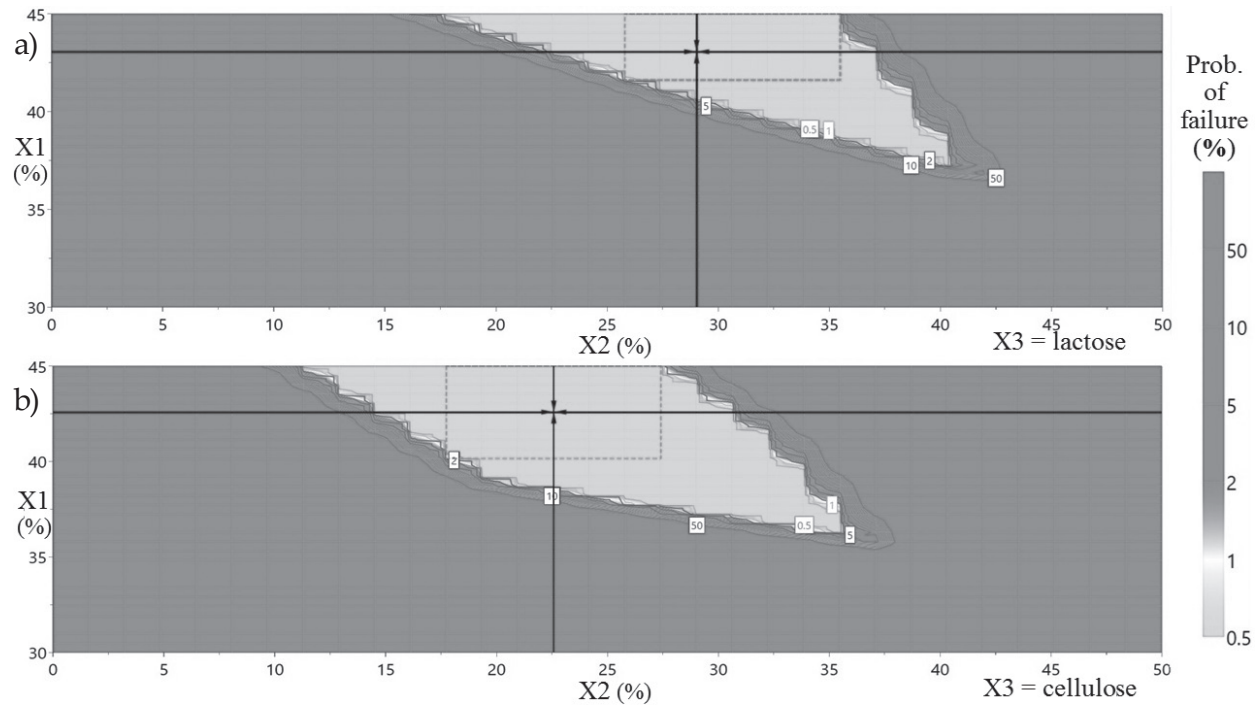

Fig. 3. The design space and proven acceptable range (PAR) for the hydrophilic matrix formulations with: a) lactose monohydrate and b) microcrystalline cellulose as diluents. $X_{1}$ - total HPMC percent; $X_{2}$ - HPMC K4M proportion of the total HPMC percent; $X_{3}$ - type of extragranular diluent.

diluent), two design spaces were generated, one for each variable. The green area represents the design space and each point from this area predicts, with a low probability of failure under $1 \%$, a possible hydrophilic matrix formulation that would possess the described CQAs (20). The dotted frame inside the design space is the design space hypercube, which defines the proven acceptable range (PAR). Within this area, factor values can vary independently from each other, while the obtained product will meet the specified quality target. The cross-hairs that can be noticed in the figures indicate the robust setpoint, representing the input values that will predict, with the highest statistical values, a result within specifications. The main difference between robust and optimal setpoints is that the optimal setpoint is focused on finding a solution as close to the target as possible (19).

Robust setpoints and hypercube edges for the two design spaces can be seen in Fig. 3; the calculated setpoint values were $X_{1}=43.06, X_{2}=29.03$ for the formulation with lactose as extragranular diluent, and $X_{1}=42.58, X_{2}=22.58$ for the formulation with microcrystalline cellulose as extragranular diluent. By analyzing Fig. 3 , it can be noticed that both the design space and the PAR are wider when using cellulose as extragranular diluent.

The following step was to use the optimization module of the software to identify optimal formulation factors based on CQAs. The levels of these factors, as well as the predicted and the obtained experimental dissolution values are shown in the first part of Table IV. Optimal formulations were performed and analyzed under the same conditions as all other experimental formulations and the obtained experimental values were very close to the theoretical ones, predicted by the experimental design.

To confirm the validity of the calculated model, a negative formulation $\left(X_{1}=30, X_{2}=\right.$ $10 X_{3}=$ microcrystalline cellulose) with values outside the design space was prepared and 
evaluated. The developed design space and the dissolution profile of all the validation formulations and of Seroquel XR are illustrated in Fig. 4. It is noticeable that the drug is released faster from the negative formulation, with different kinetics than from Seroquel $\mathrm{XR}$, all intermediate dissolution points being outside the design space, while both ideal formulations have all the dissolution points inside the design space, very close to the optimal values.

One way to compare the dissolution profile of the developed formulations and the original product was to calculate and analyze the release kinetics. As shown in the second part of Table IV, the kinetic drug release of Seroquel XR was best described by a Peppas model. The two optimal formulations had very similar release kinetics to the reference product, but were better described by zero order kinetics. The strategy of using a blend of high and low viscosity grades of HPMC has been reported before in order to achieve a zero order release profile from a matrix formulation and for reducing the variability of drug release $(30,31)$.

For evaluation of the similarities between dissolution profiles of the developed formulations and the original product, the similarity factor $f_{2}$ was used. In order to consider the two compared profiles as similar, the value of this factor needs to be greater than 50 (32). For the two optimal formulations, no significant differences in dissolution profiles were observed, the values registered for $f_{2}$ being 83.74 for the lactose formulation and 74.53 for the formulation developed with cellulose as diluent. On the other hand, the negative formulation failed the similarity test, registering an $f_{2}$ value of 49.82 .

Thus, by choosing adequately the critical formulation factors as independent variables of the experimental design and using the optimization module of the Modde 11.0 software, the theoretical optimal formulations of sustained release tablets were determined with great accuracy.

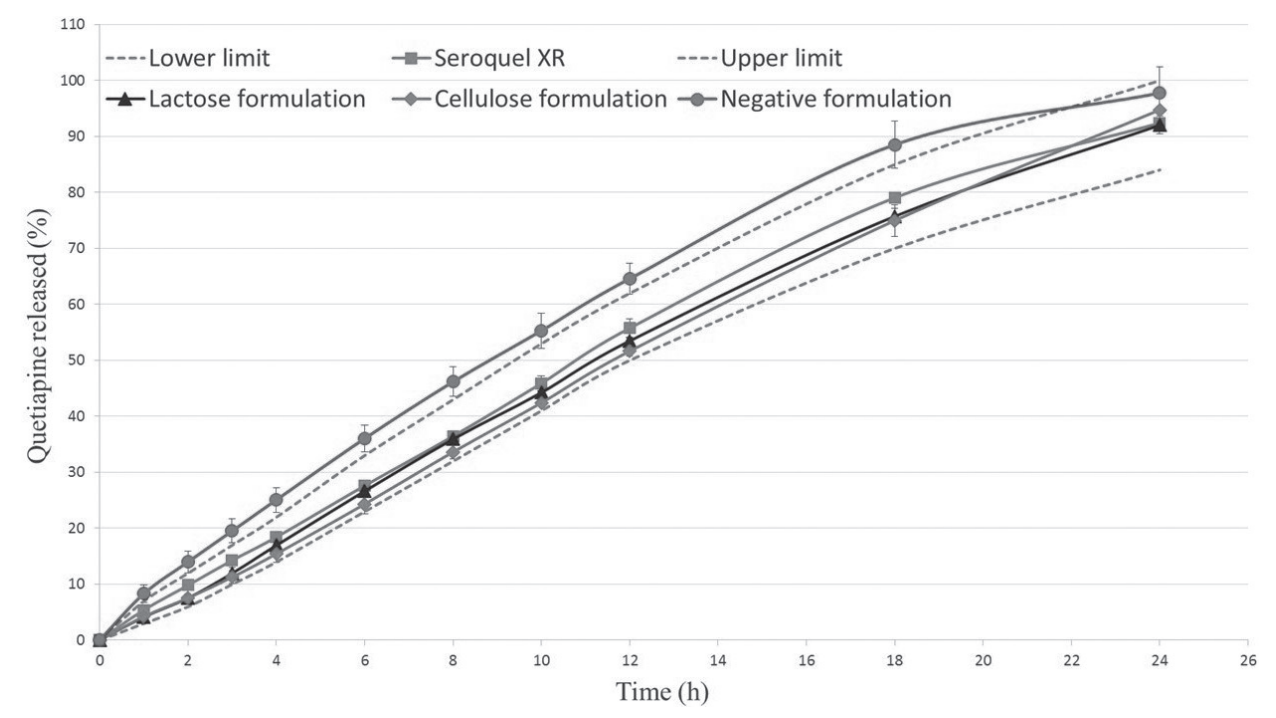

Fig. 4. Dissolution profiles of the negative and the two optimal formulations compared to Seroquel XR. 


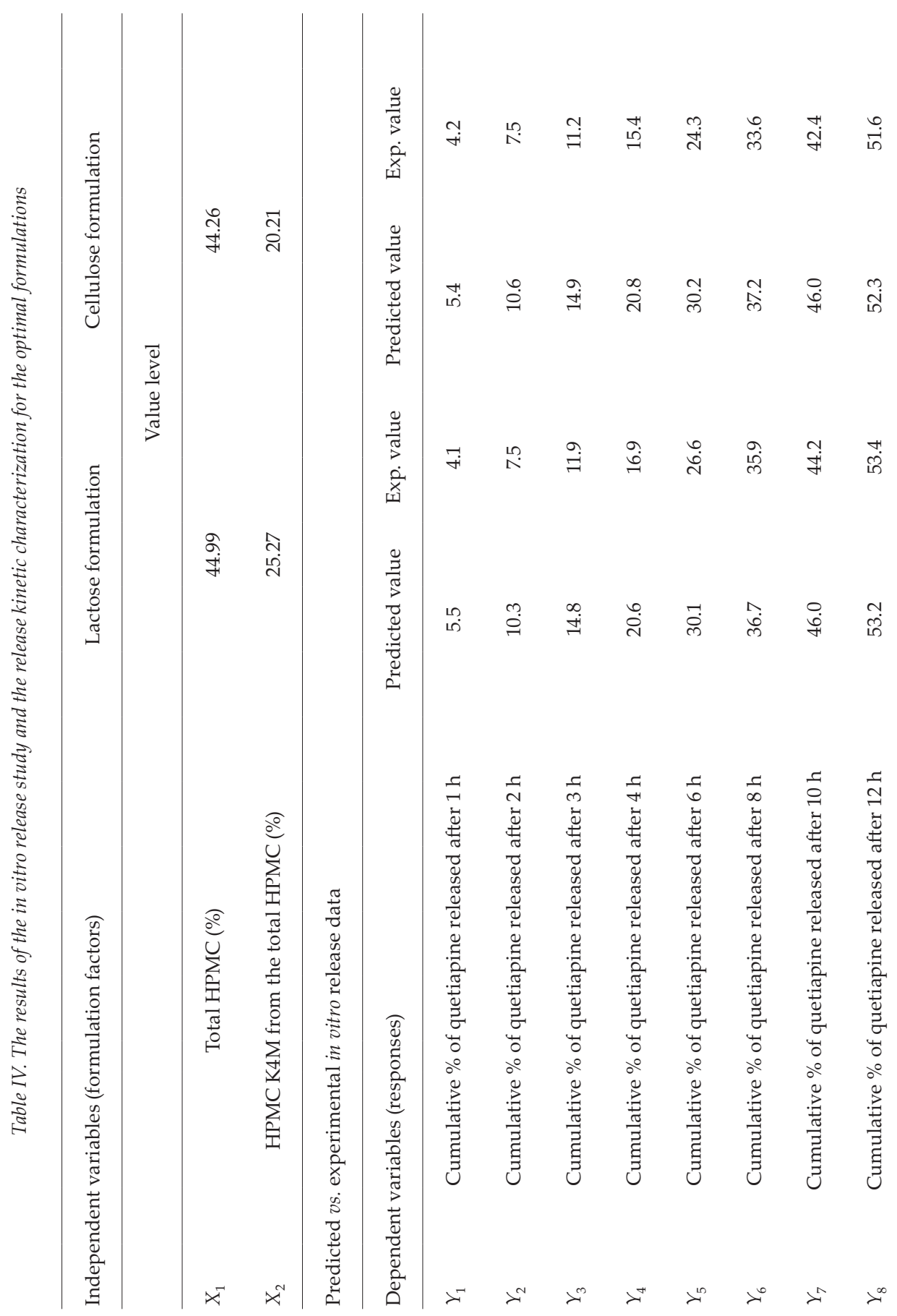




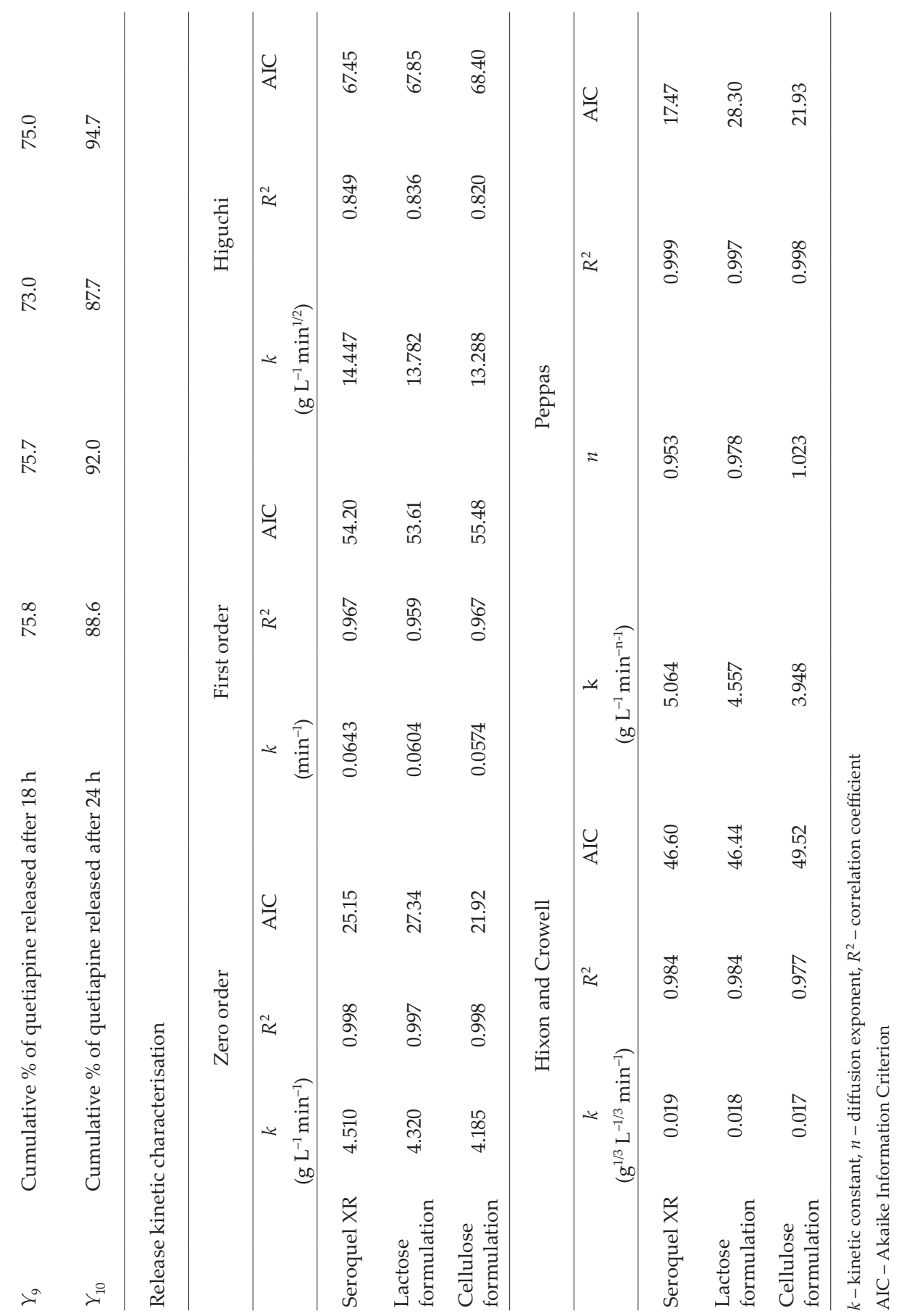


However, according to the recent studies of Kulinowski et al. $(33,34)$, in order to completely understand the hydrating process of the matrix and the release mechanisms, it would be essential to distinguish between the two key factors, namely the biopharmaceutical characteristics of the sustained release dosage form and the intrinsic properties of the matrix. The in vitro dissolution test offers valuable information regarding the biopharmaceutical properties of the sustained release dosage form and allows a good description of these characteristics, but can only deliver an indirect and incomplete characterization of the intrinsic physicochemical, structural and morphological matrix properties. In order to completely understand and assess the internal properties of the hydrophilic matrix, application of complementary techniques such as magnetic resonance imaging or micro-CT would be highly recommended.

\section{CONCLUSIONS}

The $\mathrm{QbD}$ approach has been used in this study as a strategy for the design of a sustained release tablet formulation, with a desired in vitro drug release behavior, in order to minimize the fluctuations of drug level in the blood stream, maintain the therapeutic level of the active substance in the blood, improve drug efficacy, increase patient compliance and lower the risk of side effects. Use of DoE software enabled the development and evaluation of hydrophilic matrix type sustained release formulations, as well as the observation of formulation related variable effects on the selected responses. Both studied quantitative factors proved to have important influences on the kinetic release profiles of the tablets.

The design space and optimal formulations were defined taking the drug release kinetics of Seroquel XR ${ }^{\mathrm{TM}} 200 \mathrm{mg}$ as a model. Three optimized formulations (two inside and one outside the design space) were tested in order to validate the model. The obtained results were in good accord with the predicted values, the dissolution profiles of the validation samples being similar to the targeted dissolution profile.

This study has proven that the QbD approach allows quick finding of a formulation having a dissolution profile similar to the innovative product, using a D-optimal experimental design to analyze the influence of formulation factors on the in vitro dissolution behavior.

Acknowledgement. - This work was supported by a grant of the Romanian National Authority for Scientific Research and Innovation, CNCS - UEFISCDI, project number PN-II-RU-TE-2014-4-1862.

\section{REFERENCES}

1. D. Sahu and A. Rana, Formulation development of Quetiapine Fumarate SR matrix tablets, Der Pharm. Sin. 1 (2010) 48-57.

2. S. Cheer and A. Wagstaff, Quetiapine. A review of its use in the management of schizophrenia, CNS Drugs 18 (2004) 173-199; DOI: 10.2165/00023210-200418030-00004.

3. G. Garbacz, A. Kandzi, M. Koziolek, J. Mazgalski and W. Weitschies, Release characteristics of quetiapine fumarate extended release tablets under biorelevant stress test conditions, AAPS PharmSciTech 15 (2014) 230-236; DOI: 10.1208/s12249-013-0050-2. 
A. Gavan et al:: Formulation and pharmaceutical development of quetiapine fumarate sustained release matrix tablets using a QbD approach, Acta Pharm. 67 (2017) 53-70.

4. S. Chopra, G. V. Patil and S. K. Motwani, Release modulating hydrophilic matrix systems of losartan potassium: Optimization of formulation using statistical experimental design, Eur. J. Pharm. Biopharm. 66 (2007) 73-82; DOI: 10.1016/j.ejpb.2006.09.001.

5. P. W. S. Heng, L. W. Chan, M. G. Easterbrook and X. Li, Investigation of the influence of mean HPMC particle size and number of polymer particles on the release of aspirin from swellable hydrophilic matrix tablets, J. Control. Release 76 (2001) 39-49; DOI: 10.1016/S0168-3659(01)00410-2.

6. S. Tiwari and A. R. Rajabi-Siahboomi, Modulation of drug release from hydrophilic matrices, Pharm. Tech. Eur. 20 (2008) 24-32.

7. I. Tomuta and S. E. Leucuta, The influence of formulation factors on the kinetic release of metoprolol tartrate from Prolong release coated minitablets, Drug Dev. Ind. Pharm. 33 (2007) 1070-1077; DOI: $10.1080 / 03639040601180002$.

8. R. Asija, S. Asija and M. Carpenter, Design of oral sustain release drug delivery of quetiapine fumarate, J. Drug Discov. Ther. 2 (2014) 7-11.

9. S. Tiwari and A. Rajabi-Siahboomi, Applications of complementary polymers in HPMC hydrophilic extended release matrices, Drug Deliv. Technol. 9 (2009) 20-27.

10. J. Djuriš, D. Medarević, M. Krstić, I. Vasiljević, I. Mašić and S. Ibrić, Design space approach in optimization of fluid bed granulation and tablets compression process, Sci. World J. (2012) 1-10; DOI: $10.1100 / 2012 / 185085$.

11. F. Rose, J. E. Wern, P. T. Ingvarsson, M. Van De, P. Andersen, F. Follmann and C. Foged, Engineering of a novel adjuvant based on lipid-polymer hybrid nanoparticles: A quality-by-design approach, J. Control. Release 28 (2015) 48-57; DOI: 10.1016/j.jconrel.2015.05.004.

12. L. X. Yu, Pharmaceutical quality by design: Product and process development, understanding and control, Pharm. Res. 25 (2008) 781-791; DOI: 10.1007/s11095-007-9511-1.

13. A. L. Vonica-Gligor, I. Tomuță and S. E. Leucuța, Piecewise function parameters as responses of the design of experiment in the development of a pulsatile release chronopharmaceutical system, Acta Pol. Pharm. 66 (2016) 173-189; DOI: 10.1515/acph-2016-0025.

14. S. Dash, P. N. Murthy, L. Nath and P. Chowdhury, Kinetic modeling on drug release from controlled drug delivery systems, Acta Pol. Pharm. 67 (2010) 217-223; DOI: 10.1016/S0928-0987(01)000951.

15. International Conference on Harmonisation of Technical Requirements for Registration of Pharmaceuticals for Human Use. 2009. ICH harmonised tripartite guideline: Pharmaceutical development Q8(R2); Retrieved from http://www.ich.org/fileadmin/Public_Web_Site/ICH_Products/ Guidelines/Quality/Q8_R1/Step4/Q8_R2_Guideline.pdf

16. N. Charoo, A. Shamsher, A. Zidan and Z. Rahman, Quality by design approach for formulation development: A case study of dispersible tablets, Int. J. Pharm. 423 (2012) 167-178; DOI: 10.1016/j. ijpharm.2011.12.024.

17. European Pharmacopeia 8.8. Online Edition 2016.

18. S. Shahi, D. Magar, T. Ingale and H. Mene, QbD approach in formulation and evaluation of duloxetine Hcl bilayer buccal tablets, Lit. J. Pharm. Drug Deliv. Technol. 01 (2015) 29-36; DOI: 10.15272/ ljpddt.v1i1.8.

19. MODDE 11.0. Software for Design of Experiments: User's Guide and Tutorial, Umetrics Academy, Umea 2015.

20. S. Iurian, I. Tomuta, C. Bogdan, L. Rus, T. Tokes, M. Achim, M. Moldovan and S. Leucuta, Defining the design space for freeze-dried orodispersible tablets with meloxicam, Drug Dev. Ind. Pharm. 9045 (2016); DOI: 10.1080/03639045.2016.1188108.

21. J. Wagner, Interpretation of percent dissolved-time plots derived from in vitro testing of conventional tablets and capsules, J. Pharm. Sci. 58 (1969) 1253-1257; DOI: 10.1002/jps.2600581021. 
22. I. Prasacu, C. Mircioiu, R. Sandulovici and F. Enache, Release of metoprolol from solid dosage forms. Choice and validation of theoretical model, Farmacia 57 (2009) 89-100.

23. T. Higuchi, Mechanism of sustained-action medication. Theoretical analysis of rate of release of solid drugs dispersed in solid matrices, J. Pharm. Sci. 52 (1963) 1145-1149.

24. A. W. Hixson and J. H. Crowell, Dependence of reaction velocity upon surface and agitation, Ind. Eng. Chem. 23 (1931) 923-931.

25. W. Korsmeyer, R. Gurny, E. Doelker, P. Buri and N. Peppas, Mechanisms of solute release from porous hydrophilic polymers, Int. J. Pharm. 15 (1983) 25-35; DOI: 10.1016/0378-5173(83)90064-9.

26. L. Eriksson, E. Johansson, N. Kettaneh-Wold, C. Wikstrom and S. Wold, Design of Experiments, Principles and Applications (3rd ed.), Umetrics Academy, Umea 2008.

27. A. Porfire, I. Tomuta, D. Muntean, L. Luca, E. Licarete, M. C. Alupei, M. Achim, L. Vlase and M. Banciu, Optimizing long-circulating liposomes for delivery of simvastatin to C26 colon carcinoma cells, J. Liposome Res. (2014) 1-9; DOI: 10.3109/08982104.2014.987787.

28. M. Levina and A. R. Rajabi-Siahboomi, The influence of excipients on drug release from hydroxypropyl methylcellulose matrices, J. Pharm. Sci. 93 (2004) 2746-2754; DOI: 10.1002/jps.20181.

29. R. Rowe, P. Sheskey and M. Quinn, Handbook of Pharmaceutical Excipients (6th ed.), London: Pharmaceutical Press; DOI: 10.1016/S0168-3659(01)00243-7.

30. C. Lui, Sustained Release with High and Low Viscosity HPMC, U.S. Pat. 5009895, 23 Apr 1991.

31. J. Manjusha, G. Ganesh Vinayak and M. Satish Ramanlal, Sustained Release Formulation of Alprazolam, WO2007010369 A2, 25 Jan 2007.

32. V. P. Shah, Y. Tsong, P. Sathe and J.-P. Liu, In vitro dissolution profile comparison - statistics and analysis of the similarity factor, f2 15 (1998) 889-896; DOI: 10.1023/A:1011976615750.

33. P. Kulinowski, K. Woyna-Orlewicz, J. Obrał, G. M. Rappen, D. Haznar-Garbacz, W. P. Węglarz, R. Jachowicz, G. Wyszogrodzka, J. Klaja and P. P. Dorozyński, Multimodal approach to characterization of hydrophilic matrices manufactured by wet and dry granulation or direct compression methods, Int. J. Pharm. 499 (2016) 263-270; DOI: 10.1016/j.ijpharm.2015.12.067.

34. P. Kulinowski, K. Woyna-Orlewicz, G. M. Rappen, D. Haznar-Garbacz, W. P. Weglarz and P. P. Dorozynski, An understanding of modified release matrix tablets behavior during drug dissolution as the key for prediction of pharmaceutical product performance - Case study of multimodal characterization of quetiapine fumarate tablets, Int. J. Pharm. 484 (2015) 235-245; DOI: 10.1016/j.ijpharm.2015.02.040. 